\title{
Inequalities in Products of Minors of Totally Nonnegative Matrices
}

MARK SKANDERA

mark.skandera@dartmouth.edu

Department of Mathematics, Dartmouth College, 6188 Bradley Hall, Hanover, NH 03755-3551, USA

Received January 17, 2003; Revised March 25, 2003; Accepted October 1, 2003

Abstract. Let $\Delta_{I, I^{\prime}}$ be the minor of a matrix which corresponds to row set $I$ and column set $I^{\prime}$. We give a characterization of the inequalities of the form

$$
\Delta_{I, I^{\prime}} \Delta_{K, K^{\prime}} \leq \Delta_{J, J^{\prime}} \Delta_{L, L^{\prime}}
$$

which hold for all totally nonnegative matrices. This generalizes a recent result of Fallat, Gekhtman, and Johnson.

Keywords: nonnegative matrices, inequalities of products

\section{Introduction}

Let $A$ be an $n \times n$ matrix and let $I$ and $I^{\prime}$ be subsets of $[n]=\{1, \ldots, n\}$ having equal cardinality. We define $\Delta_{I, I^{\prime}}$, the $\left(I, I^{\prime}\right)$ minor of $A$, to be the determinant of the submatrix of $A$ corresponding to rows $I$ and columns $I^{\prime}$. A matrix is called totally nonnegative if each of its minors is nonnegative. While this definition may be applied to nonsquare matrices, we will restrict our attention to square totally nonnegative matrices. It is easy to see that the concatenation of a row or column of zeros to a totally nonnegative matrix introduces no negative minors.

One setting in which totally nonnegative matrices arise is in the counting of paths in directed graphs. Let us define a planar network of order $n$ to be a planar acyclic directed graph $G=(V, E)$ in which $2 n$ vertices are distinguished as $n$ sources and $n$ sinks. We will assume that all sources and sinks are boundary vertices, labeled cyclically (counterclockwise) as $s_{1}, \ldots, s_{n}, t_{n}, \ldots, t_{1}$. We will use $S$ and $T$ to denote the sources and sinks of a planar network, and $S_{I}$ and $T_{I}$ to denote the subsets of sources and sinks corresponding to an index set $I$,

$$
\begin{aligned}
& S_{I}=\left\{s_{i} \mid i \in I\right\}, \\
& T_{I}=\left\{t_{i} \mid i \in I\right\} .
\end{aligned}
$$

In figures we will draw sources on the left of a planar network and sinks on the right. The orientations of edges will be understood to be from left to right (see figure 1). 


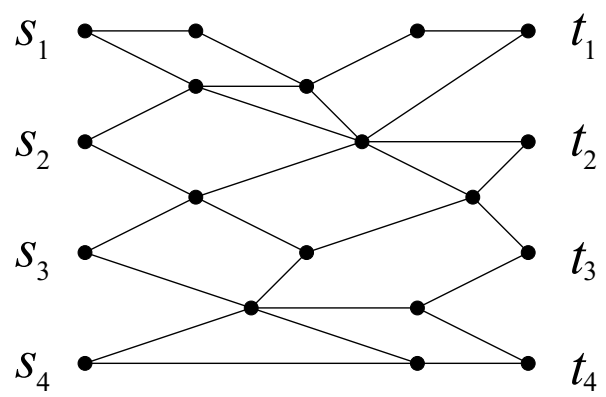

Figure 1. A planar network of order 4.

Let $G=(V, E)$ be a planar network of order $n$ and let each edge $e$ of $G$ be labeled by a positive real weight $w_{e}$. Define the weight of a path from $s$ to $t$ to be product of weights of edges along this path, and define the weighted path matrix of $G$ to be the matrix $A=\left[a_{i j}\right]$, where $a_{i j}$ is the sum of weights of all paths from source $s_{i}$ to sink $t_{j}$. Such a weighted path matrix is always totally nonnegative. In particular, the minors of such a matrix have an interpretation in terms of families $\pi=\left(\pi_{1}, \ldots, \pi_{n}\right)$ of paths in $G$ from sources to sinks. Defining the weight of a path family to be the product of weights of its $n$ paths, we have the following result.

Theorem 1.1 Let A be the weighted path matrix of a planar network $G$. The minor $\Delta_{I, I^{\prime}}$ of $A$ is equal to the sum of weights of all nonintersecting path families which connect the sources indexed by $I$ to the sinks indexed by $I^{\prime}$. In particular, A is totally nonnegative.

The first proofs of this fact were given by Karlin and MacGregor [7], and Lindström [8]. Since then, Theorem 1.1 has been used to prove that matrices arising in various situations are totally nonnegative (see for example [5] and [6]).

By results of Whitney [12], Loewner [9], Cryer [2], and Brenti [1], the converse of Theorem 1.1 is true as well. This result was first stated in [1].

Theorem 1.2 Every $n \times n$ totally nonnegative matrix $A$ is the weighted path matrix of a planar network $G$ of order $n$.

While the planar network $G$ in Theorem 1.2 is not uniquely determined, it is easy to see that $G$ may be chosen so that each source (sink) has indegree zero and outdegree one (outdegree zero and indegree one). Furthermore, it may always be chosen to be of a canonical form [1] (see also [4]).

Some recent interest in totally nonnegative matrices involves polynomial functions in $n^{2}$ variables $\left\{x_{i j} \mid i, j \in[n]\right\}$ which evaluate to nonnegative numbers whenever we set $x_{i j}=a_{i j}$ for a totally nonnegative matrix $A=\left[a_{i j}\right]$ of size at least $n \times n$. Let us call such polynomials totally nonnegative. In particular, Lusztig [10] has shown that the elements of the dual canonical basis of the coordinate ring of $G L_{n}$ are totally nonnegative. In order to 


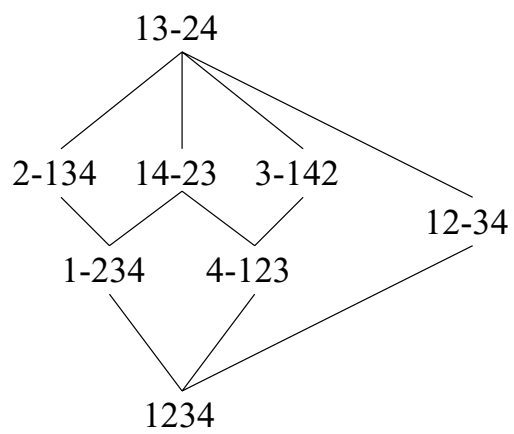

Figure 2. A partial order on partitions of $\{1,2,3,4\}$ into at most two blocks.

better understand this basis, which currently has no simple description, one might hope to characterize all totally nonnegative polynomials, or at least to study some subset of these polynomials.

One such subset, discovered recently by Fallat et al. [3], may be described in terms of principal minors. An example is the totally nonnegative polynomial

$$
\Delta_{\{1,3\}\{1,3\}} \Delta_{\{2,4\}\{2,4\}}-\Delta_{\{1,4\}\{1,4\}} \Delta_{\{2,3\}\{2,3\}} .
$$

In other words, the inequality

$$
\Delta_{\{1,4\}\{1,4\}} \Delta_{\{2,3\}\{2,3\}} \leq \Delta_{\{1,3\}\{1,3\}} \Delta_{\{2,4\}\{2,4\}}
$$

holds for all totally nonnegative matrices of size at least $4 \times 4$. This and sixteen similar inequalities are shown as a poset $P$ in figure 2. Each element of the form $I-K$ in $P$ represents the product $\Delta_{I, I} \Delta_{K, K}$, and the minimal element represents the determinant $\Delta_{\{1,2,3,4\}}$. The relation $<_{P}$ is defined by $I-K<_{p} J-L$ whenever the inequality

$$
\Delta_{I, I} \Delta_{K, K} \leq \Delta_{J, J} \Delta_{L, L}
$$

holds for all totally nonnegative matrices. Thus the relation $14-23<_{P} 13-24$ represents the inequality (1.2).

This poset raises the question of finding a more general class of inequalities in products of arbitrary (i.e., not necessarily principal) minors of totally nonnegative matrices. Let us consider products of the form $\Delta_{I, I^{\prime}} \Delta_{\bar{I}, \bar{I}^{\prime}}$, where $\bar{I}=[n] \backslash I$.

Question 1.1 What conditions on four subsets $I, I^{\prime}, J, J^{\prime}$ of $[n]$ imply the inequality

$$
\Delta_{I, I^{\prime}} \Delta_{\bar{I}, \bar{I}^{\prime}} \leq \Delta_{J, J^{\prime}} \Delta_{\bar{J}, \bar{J}^{\prime}}
$$

for all totally nonnegative matrices of size at least $n \times n$ ? 
By Theorem 1.2, we may interpret the products of minors which occur in the inequality (1.3) in terms of families of paths in planar networks. The combinatorial interpretation of the product $\Delta_{I, I^{\prime}} \Delta_{\bar{I}, \bar{I}^{\prime}}$ is quite simple.

Observation 1.3 Let $A$ be an $n \times n$ totally nonnegative matrix, and let $G$ be any planar network whose weighted path matrix is $A$. Then the product $\Delta_{I, I^{\prime}} \Delta_{\bar{I}, \bar{I}^{\prime}}$ of minors of $A$ is equal to the weighted sum of all path families $\pi=\left(\pi_{1}, \ldots, \pi_{n}\right)$ in $G$ with the following properties.

1. Each path connects a source in $S_{I}$ to a sink in $T_{I^{\prime}}$ or a source in $S_{\bar{I}}$ to a sink in $T_{\bar{I}^{\prime}}$.

2. The paths from $S_{I}$ to $T_{I^{\prime}}$ are pairwise vertex disjoint, as are the paths from $S_{\bar{I}}$ to $T_{\bar{I}^{\prime}}$.

We will refer to the combination of the two conditions in Observation 1.3 as a binary crossing rule or more specifically as the $\left(I, I^{\prime}\right)$ crossing rule. We will refer to source-to-sink paths and to families of these simply as paths and path families.

Using Observation 1.3 we may reformulate Question 1.1.

Question 1.2 What conditions on four subsets $I, I^{\prime}, J, J^{\prime}$ of $[n]$ imply that for each planar network $G$ of order n, the weighted sum of path families in $G$ which obey the $\left(I, I^{\prime}\right)$ crossing rule is less than or equal to the weighted sum of path families in $G$ which obey the $\left(J, J^{\prime}\right)$ crossing rule?

In Section 2 we will examine a special case of Question 1.2 which will lead to a proof of our main theorem in Section 3. This theorem, which generalizes recent results of Fallat et al. [3], characterizes all inequalities of the form

$$
\Delta_{I, I^{\prime}} \Delta_{\bar{I}, \bar{I}^{\prime}} \leq \Delta_{J, J^{\prime}} \Delta_{\bar{J}, \bar{J}^{\prime}}
$$

which hold for all totally nonnegative matrices. A corollary provides a combinatorial interpretation of the corresponding totally nonnegative polynomials

$$
\Delta_{J, J^{\prime}} \Delta_{\bar{J}, \bar{J}^{\prime}}-\Delta_{I, I^{\prime}} \Delta_{\bar{I}, \bar{I}^{\prime}}
$$

In Section 4 we will show that our main result essentially characterizes even the more general class of inequalities of the form

$$
\Delta_{I, I^{\prime}} \Delta_{K, K^{\prime}} \leq \Delta_{J, J^{\prime}} \Delta_{L, L^{\prime}}
$$

which hold for all totally nonnegative matrices.

\section{Path families which cover a planar network}

A path family $\pi$ in a planar network $G$ will not in general use all of the edges of $G$. We will say that $\pi$ covers $G$ if it does use all of the edges. Since the weight of a path family 
which covers $G$ is equal to the product of all edge weights in $G$ (with multiplicities for multiply covered edges), we may calculate the weighted sum of path families which cover $G$ simply by counting path families which cover $G$. This suggests the following specialization of Question 1.2.

Question 2.1 What conditions on four subsets $I, I^{\prime}, J, J^{\prime}$ of $[n]$ imply that for each planar network $G$ of order $n$, the number of path families which cover $G$ and obey the $\left(I, I^{\prime}\right)$ crossing rule is less than or equal to the number of path families which cover $G$ and obey the $\left(J, J^{\prime}\right)$ crossing rule?

Questions 1.2 and 2.1 in fact have the same answer. The easiest way to see this is to consider three related families of graphs and maps between these.

Let $\mathcal{G}_{1}(n)$ be the family of planar networks of order $n$ which may be expressed as a union of $n$ source-to-sink paths, no three of which share a vertex. These are precisely the planar networks which can be covered by path families which obey a binary crossing rule. (To see sufficiency, let $I=I^{\prime}$ be the set of odd integers in $[n]$.) Note that the vertices in such planar networks have indegree and outdegree bounded by two.

Let $\mathcal{G}_{2}(n)$ be the family of undirected graphs with at least $n$ connected components which are paths, and arbitrarily many connected components which are cycles. To define our first map $\phi: \mathcal{G}_{1}(n) \rightarrow \mathcal{G}_{2}(n)$, let $G=(V, E)$ be a graph in $\mathcal{G}_{1}$, and create $\phi(G)=G^{\prime}=\left(V^{\prime}, E^{\prime}\right)$ as follows.

1. For each vertex $x \in V$ which has indegree and/or outdegree two, create vertices $x^{-}$and $x^{+}$in $V^{\prime}$. Otherwise create vertex $x$ in $V^{\prime}$.

2. For each edge $(x, y)$ in $E$, create the unique edge $\left(x^{+}, y^{-}\right)$or $\left(x, y^{-}\right)$or $\left(x^{+}, y\right)$ or $(x, y)$ which can be defined in $E^{\prime}$.

Figure 3 shows a planar network $G$ and figure 4 shows the graph $\phi(G)$. As we have used $S$ and $T$ to refer to the sources and sinks in $G$, we will again use $S$ and $T$ to refer to the corresponding vertices in $\phi(G)$.

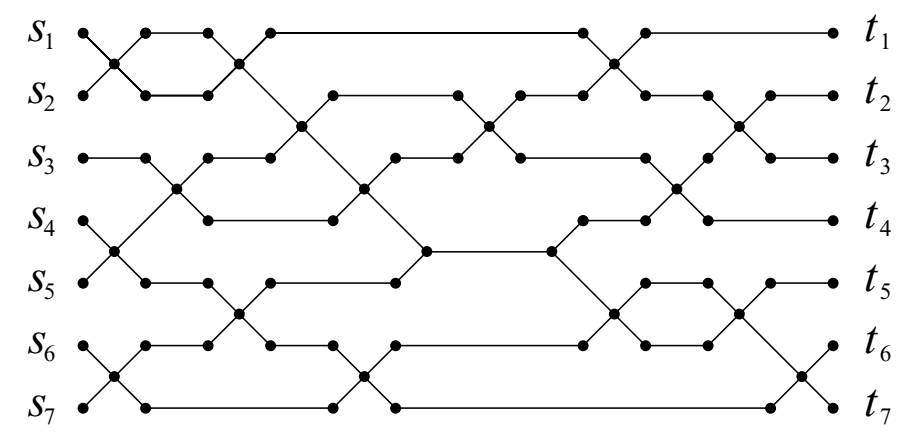

Figure 3. A planar network $G$ which is a union of 7 paths. 


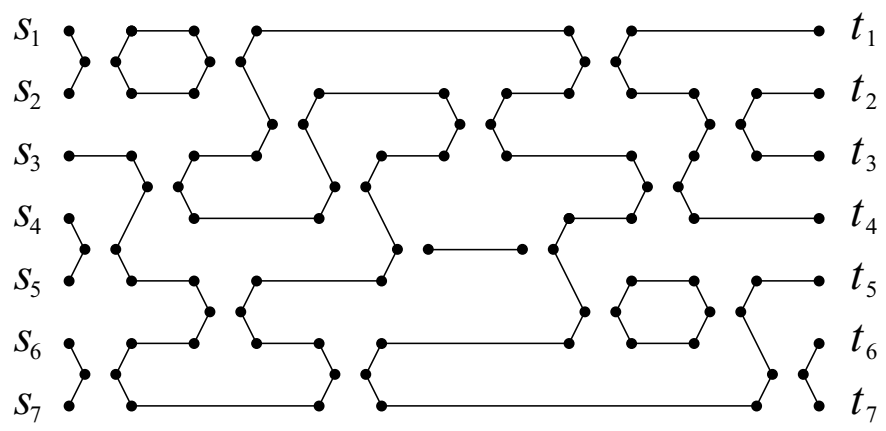

Figure 4. The graph $\phi(G)$.

It is not hard to see that the components of $\phi(G)$ are of three types: paths whose two endvertices are sources or sinks (there are necessarily $n$ of these), paths containing no source or sink, and cycles. Examination of these components immediately gives a formula for the number of path families which cover $G$ and obey a binary crossing rule.

Proposition 2.1 Let $G$ be a planar network in $\mathcal{G}_{1}(n)$, let $k$ be the number of cyclic components of $\phi(G)$, and let $I, I^{\prime}$ be two subsets of $[n]$. Then the number of path families which cover $G$ and obey the $\left(I, I^{\prime}\right)$ crossing rule is $2^{k}$ if no path component of $\phi(G)$ contains two vertices in $S_{I} \cup T_{\bar{I}^{\prime}}$. It is zero otherwise.

Proof: Let $G$ be a graph in $\mathcal{G}_{1}(n)$. Counting the number of path families which cover $G$ and obey the $\left(I, I^{\prime}\right)$ crossing rule is equivalent to counting the edge colorings of $G$ which satisfy the following conditions.

1. Each edge incident upon a source in $S_{I}$ or a sink in $T_{I^{\prime}}$ is colored blue; edges incident upon the remaining sources and sinks are colored red.

2. Each edge in the graph must be colored red, blue, or red and blue.

3. For each vertex $v$ which is not a source or a sink, at most one red edge enters $v$ and the same number of red edges leaves $v$.

4. For each vertex $v$ which is not a source or a sink, at most one blue edge enters $v$ and the same number of blue edges leaves $v$.

These rules ensure that $|I|$ nonintersecting blue paths lead from $S_{I}$ to $T_{I^{\prime}}, n-|I|$ nonintersecting red paths lead from $S_{\bar{I}}$ to $T_{\bar{I}^{\prime}}$, and the union of these $n$ paths is equal to the edge set of $G$.

Consider any component $H$ of $\phi(G)$ and let $F$ be the corresponding set of edges in $G$. For any two edges in $H$ which share a vertex $x^{+}$or $x^{-}$, the corresponding edges of $F$ must be colored differently. Similarly, for any two edges in $H$ which share a vertex $x$, the corresponding edges in $F$ must be colored equally. This forces us to color $F$ by partitioning it into alternating blocks of equally colored edges, with consecutive blocks separated by a vertex of the form $x^{+}$or $x^{-}$. 
If $H$ is a path containing no vertex in $S \cup T$, then we must color each edge in $F$ both red and blue, since the unique vertex $x^{+}$in $H$ corresponds to a vertex $x$ in $G$ with indegree two. If $H$ is a cycle, then it contains equal numbers of vertices of the forms $x^{+}$and $x^{-}$, and contains no sources or sinks. Thus we partition $F$ into an even number of monochromatic blocks, and we may color this set of blocks in one of two ways. Now suppose $H$ is a path whose endvertices belong to $S \cup T$. If the endvertices both belong to $S$ or both to $T$, then $H$ contains an odd number of vertices of the forms $x^{+}, x^{-}$, and we partition $F$ into an even number of blocks. Thus we may color this set of blocks in one way if exactly one of the endvertices of $H$ belongs to $S_{I} \cup T_{I^{\prime}}$ and in zero ways otherwise. If on the other hand one endvertex of $H$ belongs to $S$ and the other belongs to $T$, then $H$ contains an even number of vertices of the forms $x^{+}, x^{-}$and we partition $F$ into an odd number of blocks. Thus we may color this set of blocks in one way if exactly one of the endvertices of $H$ belongs to $S_{I} \cup T_{I^{\prime}}$ and in zero ways otherwise.

Proposition 2.1 implies a surprising relationship between sets of path families which cover a planar network and obey one crossing rule or another.

Corollary 2.2 Let $G$ be a planar network of order $n$ and let $I, I^{\prime}, J, J^{\prime}$ be subsets of $[n]$. If the number of path families which cover $G$ and obey the $\left(I, I^{\prime}\right)$ crossing rule is not equal to the number of path families which cover $G$ and obey the $\left(J, J^{\prime}\right)$ crossing rule, then one of these numbers is zero.

Thus, the comparison of weighted sums of path families which cover a planar network $G$ and obey two different crossing rules reduces to a problem of determining the existence of such families. By Proposition 2.1, this in turn reduces to the problem of deciding if $n$ paths in the graph $\phi(G)$ determine a perfect matching of $S_{I} \cup T_{\bar{I}^{\prime}}$ with $S_{\bar{I}} \cup T_{I^{\prime}}$. We therefore introduce a third class of graphs which records only this information.

Let $\mathcal{G}_{3}(2 n)$ be the family of graphs on $2 n$ vertices labeled $1, \ldots, 2 n$, and $n$ edges which can be drawn so that if the vertices lie in increasing order on a horizontal line, then the edges form $n$ noncrossing arcs above them. It is well known that the cardinality of $\mathcal{G}_{3}(2 n)$ is $C_{n}$, the $n$th Catalan number (see [11, p. 222]). To define a second map $\psi: \mathcal{G}_{1}(n) \rightarrow \mathcal{G}_{3}(2 n)$, let $G=(V, E)$ be a graph in $\mathcal{G}_{1}(n)$, let $G^{\prime}=\left(V^{\prime}, E^{\prime}\right)$ be $\phi(G)$ and create $\psi(G)=G^{\prime \prime}=$ $\left(V^{\prime \prime}, E^{\prime \prime}\right)$ as follows.

1. For each source $s_{i}$ in $V$ create vertex $i$ in $V^{\prime \prime}$.

2. For each sink $t_{i}$ in $V$ create vertex $2 n+1-i$ in $V^{\prime \prime}$.

3. For each path component of $G^{\prime}$ with endvertices in $S \cup T$, connect the corresponding two vertices in $V^{\prime \prime}$ with an arc.

Figures 5 and 6 show two embeddings of the graph $\psi(G)$, where $G$ is the graph in figure 3.

The map $\psi$ allows us to state a simple criterion for comparing path families which cover a given graph in $\mathcal{G}_{1}(n)$ and obey two different crossing rules. 


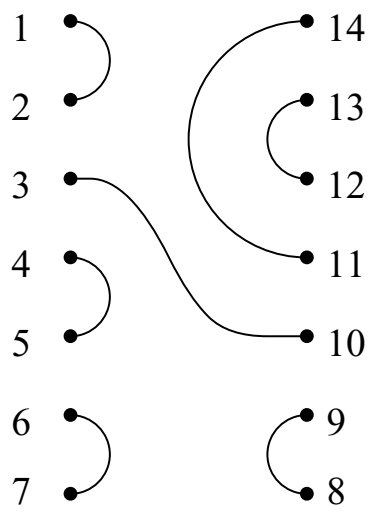

Figure 5. The graph $\psi(G)$, drawn similarly to $\phi(G)$.

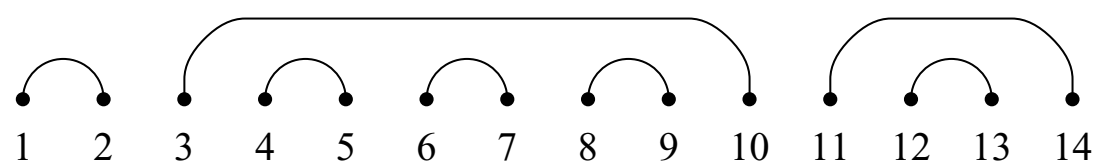

Figure 6. The graph $\psi(G)$, drawn with vertices on a horizontal line and edges above this line.

Observation 2.3 Let $G$ be a graph in $\mathcal{G}_{1}(n)$, let $I, I^{\prime}, J, J^{\prime}$ be subsets of $[n]$, and define the sets

$$
\begin{aligned}
& I^{\prime \prime}=I \cup\left\{2 n+1-i \mid i \in \bar{I}^{\prime}\right\}, \\
& \bar{I}^{\prime \prime}=[2 n] \backslash I^{\prime \prime}, \\
& J^{\prime \prime}=J \cup\left\{2 n+1-j \mid j \in \bar{J}^{\prime}\right\}, \\
& \overline{J^{\prime \prime}}=[2 n] \backslash J^{\prime \prime} .
\end{aligned}
$$

There are more path families which cover $G$ and obey the $\left(I, I^{\prime}\right)$ crossing rule than there are path families which cover $G$ and obey the $\left(J, J^{\prime}\right)$ crossing rule if and only if $\psi(G)$ is a perfect matching of the numbers $I^{\prime \prime}$ with $\bar{I}^{\prime \prime}$ and it is not a perfect matching of the numbers $J^{\prime \prime}$ with $\bar{J}^{\prime \prime}$.

It is easy to show that the map $\psi$ is surjective. One proof of this is given by the following map $\rho: \mathcal{G}_{3}(2 n) \rightarrow \mathcal{G}_{1}(n)$, which identifies a canonical representative in the preimage $\psi^{-1}(H)$ of each graph $H$ in $\mathcal{G}_{3}(2 n)$.

1. Draw a vertical representation of $H$ and label the vertices $s_{1}, \ldots, s_{n}, t_{n}, \ldots, t_{1}$ as in figure 7(a).

2. For each edge $e$ of $H$ whose endvertices have indices which differ by $k$, replace $e$ by a zig-zag path of $k+1$ straight edges and $k$ new vertices as in figure 7(b). 


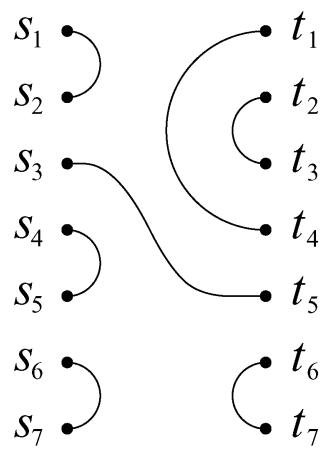

(a) $H$

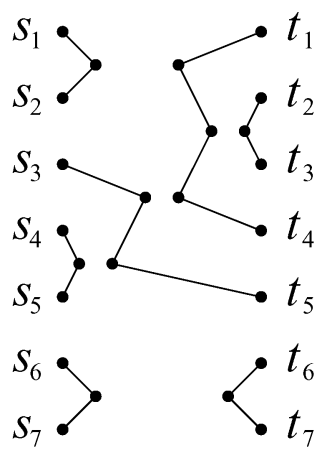

(b)

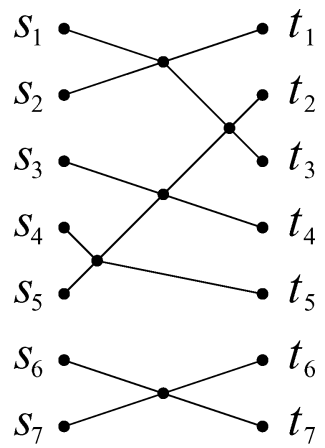

(c) $\rho(H)$

Figure 7. A graph $H$ in $\mathcal{G}_{3}(14)$ and the creation of the graph $\rho(H)$ in $\mathcal{G}_{2}(7)$.

3. Identify pairs of new vertices as in figure $7(\mathrm{c})$ so that each vertex in the resulting graph has degree one or four.

It is easy to see that the graph created in step 2 is $\phi(\rho(H))$, and that all pairs of vertices which are identified in step 3 belong to two different components of $\phi(\rho(H))$. More specifically, we have the following lemma.

Lemma 2.4 Let $H$ be a graph in $\mathcal{G}_{3}(2 n)$, and let $I, I^{\prime}$ be subsets of $[n]$. If $\rho(H)$ contains any path family which obeys the $\left(I, I^{\prime}\right)$ crossing rule, then this path family covers $\rho(H)$ and is unique.

Proof: Let $\pi$ be a path family in $\rho(H)$ which obeys the $\left(I, I^{\prime}\right)$ crossing rule but does not cover $\rho(H)$, and color the edges of $\rho(H)$ red and/or blue as in the proof of Proposition 2.1. Construct the graph $\phi(\rho(H))$, and color its edges as those of $\rho(H)$ are colored. All components of $\phi(\rho(H))$ are paths, some containing uncolored edges. For each component containing at least one uncolored edge, consider the vertex labels of the corresponding arc in $H$, and choose a component $C$ which minimizes the difference between these two labels. By the choice of $C$ and the fact that no component of $\phi(\rho(H))$ contains two vertices $x^{+}$ and $x^{-}$for any vertex $x$ of $\rho(H)$, we have that some edge of $C$ is uncolored, one of its neighboring edges is colored twice, the next edge is uncolored, etc. This implies that a terminal edge of $C$ is colored twice or not at all, contradicting our assumption that the edge coloring determines a path family in $\rho(H)$ which obeys the $\left(I, I^{\prime}\right)$ crossing rule.

\section{Main theorem}

The maps $\phi$ and $\psi$ defined in Section 2 reduce the problem of comparing products $\Delta_{I, I^{\prime}} \Delta_{\bar{I}, \bar{I}^{\prime}}$, $\Delta_{J, J^{\prime}} \Delta_{\bar{J}, \bar{J}^{\prime}}$ of minors in $n \times n$ totally nonnegative matrices to a problem of examining perfect matchings in graphs on $2 n$ vertices. We will now demonstrate that this problem reduces to 
examining subintervals of $[2 n]$ and their intersections with the two sets $I^{\prime \prime}, J^{\prime \prime}$ defined in Observation 2.3. In particular, we will define an interval $\left[b_{1}, b_{2}\right]=\left\{b_{1}, b_{1}+1, \ldots, b_{2}\right\}$ to be even if its cardinality is even, and we will show that the inequality

$$
\Delta_{I, I^{\prime}} \Delta_{\bar{I}, \bar{I}^{\prime}} \leq \Delta_{J, J^{\prime}} \Delta_{\bar{J}, \bar{J}^{\prime}}
$$

holds for all totally nonnegative matrices if and only if the sets $I^{\prime \prime}, J^{\prime \prime}$ satisfy the inequality

$$
\max \left\{\left|B \cap I^{\prime \prime}\right|,\left|B \backslash I^{\prime \prime}\right|\right\} \geq \max \left\{\left|B \cap J^{\prime \prime}\right|,\left|B \backslash J^{\prime \prime}\right|\right\}
$$

for all even subintervals $B$ of $[2 n]$.

Let us first consider the circumstances under which the inequality (3.1) fails to hold for some subinterval $B$ of $[2 n]$.

Lemma 3.1 Let $I^{\prime \prime}$ and $J^{\prime \prime}$ be two subsets of $[2 n]$ and let $B=\left[b_{1}, b_{2}\right]$ be a minimal subinterval of $[2 n]$ which satisfies

$$
\max \left\{\left|B \cap I^{\prime \prime}\right|,\left|B \backslash I^{\prime \prime}\right|\right\}<\max \left\{\left|B \cap J^{\prime \prime}\right|,\left|B \backslash J^{\prime \prime}\right|\right\} .
$$

Then the numbers $b_{1}$ and $b_{2}$ both belong to $J^{\prime \prime}$, or both belong to $\bar{J}^{\prime \prime}$, while exactly one of these numbers belongs to I. Furthermore, the cardinalities $\left|\left[b_{1}+1, b_{2}-1\right] \cap I^{\prime \prime}\right|$, $\left|\left[b_{1}+1, b_{2}-1\right] \backslash I^{\prime \prime}\right|,\left|\left[b_{1}+1, b_{2}-1\right] \cap J^{\prime \prime}\right|$, and $\left|\left[b_{1}+1, b_{2}-1\right] \backslash J^{\prime \prime}\right|$ are all equal to $\frac{1}{2}(|B|-2)$. In particular, $B$ is even.

Proof: Assume without loss of generality that we have

$$
\left|B \backslash J^{\prime \prime}\right|<\left|B \backslash I^{\prime \prime}\right| \leq\left|B \cap I^{\prime \prime}\right|<\left|B \cap J^{\prime \prime}\right| .
$$

The cardinality $\left|B \cap J^{\prime \prime}\right|$ must be exactly one greater than $\left|B \cap I^{\prime \prime}\right|$, for otherwise $B$ would not be a minimal interval satisfying the inequality (3.2). Similarly, $b_{1}$ and $b_{2}$ both belong to $J^{\prime \prime}$, while at most one of these belongs to $I^{\prime \prime}$.

If neither $b_{1}$ nor $b_{2}$ belongs to $I^{\prime \prime}$, then let $c$ be the smallest number such that the cardinalities of $\left[b_{1}, c\right] \cap I^{\prime \prime}$ and $\left[b_{1}, c\right] \backslash I^{\prime \prime}$ are equal. If in addition the cardinalities of $\left[b_{1}, c\right] \cap J^{\prime \prime}$ and $\left[b_{1}, c\right] \backslash J^{\prime \prime}$ are equal, then the interval $\left[c+1, b_{2}\right]$ satisfies an inequality of the form (3.2). If not, then the interval $\left[b_{1}, c\right]$ satisfies an inequality of this form. Either possibility contradicts the minimality of $B$.

If only one of the numbers $b_{1}, b_{2}$ belongs to $I^{\prime \prime}$, then by symmetry we may assume it is $b_{1}$. Then $\left|\left[b_{1}+1, b_{2}\right] \cap J^{\prime \prime}\right|$ is equal to the maximum of $\left|\left[b_{1}+1, b_{2}\right] \cap I^{\prime \prime}\right|$ and $\left|\left[b_{1}+1, b_{2}\right] \backslash I^{\prime \prime}\right|$. Therefore the intervals $\left[b_{1}+1, b_{2}\right]$ and $B$ satisfy

$$
\begin{aligned}
\left|\left[b_{1}+1, b_{2}\right] \cap I^{\prime \prime}\right| & =\left|B \cap I^{\prime \prime}\right|-1<\left|B \cap I^{\prime \prime}\right|=\left|B \cap J^{\prime \prime}\right|-1 \\
& =\left|\left[b_{1}+1, b_{2}\right] \cap J^{\prime \prime}\right|,
\end{aligned}
$$

and we have

$$
\left|\left[b_{1}+1, b_{2}\right] \backslash I^{\prime \prime}\right|=\left|\left[b_{1}+1, b_{2}\right] \cap J^{\prime \prime}\right|=\left|B \cap I^{\prime \prime}\right| .
$$


This implies that the cardinalities of the sets $\left[b_{1}+1, b_{2}-1\right] \cap I^{\prime \prime},\left[b_{1}+1, b_{2}-1\right] \backslash I^{\prime \prime}$, $\left[b_{1}+1, b_{2}-1\right] \cap J^{\prime \prime}$, and $\left[b_{1}+1, b_{2}-1\right] \backslash J^{\prime \prime}$ are all equal to $\frac{1}{2}(|B|-2)$.

Armed with this technical lemma, we may now state and prove our main result.

Theorem 3.2 Let $I, I^{\prime}, J, J^{\prime}$ be subsets of $[n]$ and define the subsets $I^{\prime \prime}, J^{\prime \prime}$ of $[2 n]$ by

$$
\begin{aligned}
& I^{\prime \prime}=I \cup\left\{2 n+1-i \mid i \in \bar{I}^{\prime}\right\}, \\
& J^{\prime \prime}=J \cup\left\{2 n+1-j \mid j \in \bar{J}^{\prime}\right\} .
\end{aligned}
$$

Then the following statements are equivalent.

1. In each totally nonnegative matrix of size at least $n \times n$, the minors $\Delta_{I, I^{\prime}}, \Delta_{\bar{I}, \bar{I}^{\prime}}, \Delta_{J, J^{\prime}}$, and $\Delta_{\bar{J}, \bar{J}^{\prime}}$ satisfy

$$
\Delta_{I, I^{\prime}} \Delta_{\bar{I}, \bar{I}^{\prime}} \leq \Delta_{J, J^{\prime}} \Delta_{\bar{J}, \bar{J}^{\prime}}
$$

2. In each planar network $G$ of order $n$, the weighted sum of path families in $G$ which obey the $\left(I, I^{\prime}\right)$ crossing rule is less than or equal to that of the path families in $G$ which obey the $\left(J, J^{\prime}\right)$ crossing rule.

3. For each planarnetwork $G=(V, E)$ in which the edges are weighted by formal variables $w_{1}, \ldots, w_{m}$, the difference between the weighted sum of path families in $G$ which obey the $\left(J, J^{\prime}\right)$ crossing rule and that of path families in $G$ which obey the $\left(I, I^{\prime}\right)$ crossing rule is a subtraction-free polynomial in $w_{1}, \ldots, w_{m}$.

4. For each planar network $G$ of order $n$, the number of path families which cover $G$ and obey the $\left(I, I^{\prime}\right)$ crossing rule is zero, or is equal to the number of path families which cover $G$ and obey the $\left(J, J^{\prime}\right)$ crossing rule.

5. For each even subinterval $B$ of $[2 n]$, the sets $I^{\prime \prime}$ and $J^{\prime \prime}$ satisfy

$$
\max \left\{\left|B \cap I^{\prime \prime}\right|,\left|B \backslash I^{\prime \prime}\right|\right\} \geq \max \left\{\left|B \cap J^{\prime \prime}\right|,\left|B \backslash J^{\prime \prime}\right|\right\}
$$

6. For each subinterval $B$ of $[2 n]$, the sets $I^{\prime \prime}$ and $J^{\prime \prime}$ satisfy (3.3).

Proof: $\quad(1 \Leftrightarrow 2)$ Follows from Observation 1.3.

$(3 \Rightarrow 2)$ Obvious.

( $4 \Rightarrow 3$ ) The difference between the weighted sums of path families in $G$ which obey the two binary crossing rules can be expressed as a sum of differences, over all subnetworks of $G$ which can be covered by path families which obey at least one of the two rules. Assume as in (4) that the difference corresponding to each subnetwork is a monomial in $w_{1}, \ldots, w_{m}$ with coefficient $(a-a)$ or $(a-0)$ for some nonnegative integer $a$. Clearly the sum of these is a subtraction-free polynomial in $w_{1}, \ldots, w_{m}$.

$(5 \Rightarrow 4)$ Let $G$ be a planar network, and let $k\left(I, I^{\prime}\right), k\left(J, J^{\prime}\right)$ be the numbers of path families which cover $G$ and obey the $\left(I, I^{\prime}\right)$ crossing rule and the $\left(J, J^{\prime}\right)$ crossing rule, respectively. If $k\left(I, I^{\prime}\right)$ is greater than $k\left(J, J^{\prime}\right)$, then $k\left(J, J^{\prime}\right)$ must be equal to zero by 
Corollary 2.2. Thus $\psi(G)$ is a perfect matching of $I^{\prime \prime}$ with $\bar{I}^{\prime \prime}$ and not a perfect matching of $J^{\prime \prime}$ with $\bar{J}^{\prime \prime}$. Let $B=\left[b_{1}, b_{2}\right]$ be a minimal subinterval of $[2 n]$ such that $\left(b_{1}, b_{2}\right)$ is an edge in $\psi(G)$ and $b_{1}, b_{2}$ both belong to $J^{\prime \prime}$ or both belong to $\bar{J}^{\prime \prime}$. By the minimality of $B$ we have

$$
\max \left\{\left|B \cap I^{\prime \prime}\right|,\left|B \backslash I^{\prime \prime}\right|\right\}=\frac{b_{2}-b_{1}+1}{2}<\frac{b_{2}-b_{1}+3}{2}=\max \left\{\left|B \cap J^{\prime \prime}\right|,\left|B \backslash J^{\prime \prime}\right|\right\},
$$

which contradicts (5).

$(6 \Rightarrow 5)$ Obvious.

( $2 \Rightarrow 6$ ) Suppose that (6) is false. Let $B=\left[b_{1}, b_{2}\right]$ be a minimal subinterval of $[2 n]$ which satisfies

$$
\max \left\{\left|B \cap I^{\prime \prime}\right|,\left|B \backslash I^{\prime \prime}\right|\right\}<\max \left\{\left|B \cap J^{\prime \prime}\right|,\left|B \backslash J^{\prime \prime}\right|\right\} .
$$

Without loss of generality, assume that we have

$$
\left|B \backslash J^{\prime \prime}\right|<\left|B \backslash I^{\prime \prime}\right| \leq\left|B \cap I^{\prime \prime}\right|<\left|B \cap J^{\prime \prime}\right| .
$$

By Lemma 3.1, the cardinality of $B$ is even, and exactly half of the elements of the subinterval $\left[b_{1}+1, b_{2}-1\right]$ belong to $I^{\prime \prime}$. Furthermore, both $b_{1}$ and $b_{2}$ belong to $J^{\prime \prime}$ while only one of these belongs to $I^{\prime \prime}$.

Create a graph $H$ in $\mathcal{G}_{3}(2 n)$ by drawing $2 n$ distinct points labeled $1, \ldots, 2 n$ on a horizontal line, and by connecting them with $n$ nonintersecting arcs above the line as follows.

1. Connect $b_{1}$ to $b_{2}$.

2. Below the arc $\left(b_{1}, b_{2}\right)$ and above the horizontal line, draw arcs to create a noncrossing perfect matching of $\left[b_{1}+1, b_{2}-1\right] \cap I^{\prime \prime}$ with $\left[b_{1}+1, b_{2}-1\right] \backslash I^{\prime \prime}$.

3. Above the arc $\left(b_{1}, b_{2}\right)$ and above the horizontal line, draw arcs to create a noncrossing perfect matching of $([2 n] \backslash B) \cap I^{\prime \prime}$ with $([2 n] \backslash B) \backslash I^{\prime \prime}$.

Clearly $H$ is a matching of $I^{\prime \prime}$ with $\overline{I^{\prime \prime}}$, but not a matching of $J^{\prime \prime}$ with $\overline{J^{\prime \prime}}$. Now consider the graph $G=\rho(H)$ in $\mathcal{G}_{1}(n)$. By Lemma 2.4, exactly one path family in $G$ obeys the $\left(I, I^{\prime}\right)$ crossing rule and none obeys the $\left(J, J^{\prime}\right)$ crossing rule. This contradicts (2).

As an immediate corollary of Theorem 3.2, we obtain a combinatorial interpretation of the difference

$$
\Delta_{J, J^{\prime}} \Delta_{\bar{J}, \bar{J}^{\prime}}-\Delta_{I, I^{\prime}} \Delta_{\bar{I}, \bar{I}^{\prime}},
$$

when this difference is nonnegative for all totally nonnegative matrices.

Corollary 3.3 Let $I, I^{\prime}, J$ and $J^{\prime}$ be subsets of $[n]$, define the sets $I^{\prime \prime}$ and $J^{\prime \prime}$ as in Theorem 3.2, and assume that the inequality

$$
\max \left\{\left|B \cap I^{\prime \prime}\right|,\left|B \backslash I^{\prime \prime}\right|\right\} \geq \max \left\{\left|B \cap J^{\prime \prime}\right|,\left|B \backslash J^{\prime \prime}\right|\right\} .
$$


holds for all even subintervals $B$ of $[2 n]$. Then for any $n \times n$ totally nonnegative matrix $A$, and any planar network $G$ whose weighted path matrix is $A$, the difference (3.4) is equal to the weighted sum of path families $\pi$ in $G$ which obey the $\left(J, J^{\prime}\right)$ crossing rule and which cannot be covered by any path family which obeys the $\left(I, I^{\prime}\right)$ crossing rule.

As a second corollary of Theorem 3.2, we obtain the following specialization to principal minors, first stated in [3, Thm. 4.10].

Corollary 3.4 Let I and $J$ be subsets of $[n]$. Then the following statements are equivalent. 1. In each totally nonnegative matrix of size at least $n \times n$, the principal minors $\Delta_{I, I}, \Delta_{\bar{I}, \bar{I}}$, $\Delta_{J, J}$, and $\Delta_{\bar{J}, \bar{J}}$ satisfy

$$
\Delta_{I, I} \Delta_{\bar{I}, \bar{I}} \leq \Delta_{J, J} \Delta_{\bar{J}, \bar{J}}
$$

2. For each even subinterval $B$ of $[n]$, the sets I and $J$ satisfy

$$
\max \{|B \cap I|,|B \backslash I|\} \geq \max \{|B \cap J|,|B \backslash J|\} .
$$

Proof: $\quad(1 \Rightarrow 2)$ follows from Theorem 3.2.

$(2 \Rightarrow 1)$ Suppose that $I$ and $J$ satisfy (3.7) for all even subintervals of [ $n]$, and define the subsets $I^{\prime \prime}$ and $J^{\prime \prime}$ of $[2 n]$ as before Theorem 3.2. If $I^{\prime \prime}$ and $J^{\prime \prime}$ fail to satisfy (3.3) for some even subinterval of [ $2 n]$, then this interval must be of the form $B=\left[b_{1}, 2 n+1-b_{2}\right]$, where $b_{1}, b_{2} \leq n$. We cannot have $b_{1}=b_{2}$, for then the four sets $B \cap I^{\prime \prime}, B \backslash I^{\prime \prime}, B \cap J^{\prime \prime}$, $B \backslash J^{\prime \prime}$ would all have cardinality equal to $n-b_{1}+1$. On the other hand, we cannot have $b_{1} \neq b_{2}$, for then $I$ and $J$ would fail to satisfy (3.7) for the interval whose endpoints are $b_{1}$ and $b_{2}$. Thus $I^{\prime \prime}$ and $J^{\prime \prime}$ must satisfy (3.3) and the principal minors $\Delta_{I, I}, \Delta_{\bar{I}, \bar{I}}, \Delta_{J, J}$, and $\Delta_{\bar{J}, \bar{J}}$ must satisfy (3.6).

Note the similarity between the inequalities (3.3) and (3.7). This implies that the map $\left(I, I^{\prime}, n\right) \mapsto\left(I^{\prime \prime}, 2 n\right)$ induces a bijective correspondence between inequalities

$$
\Delta_{I, I^{\prime}} \Delta_{\bar{I}, \bar{I}^{\prime}} \leq \Delta_{J, J^{\prime}} \Delta_{\bar{J}, \bar{J}^{\prime}}
$$

in products of minors of $n \times n$ matrices and inequalities

$$
\Delta_{I^{\prime \prime}, I^{\prime \prime}} \Delta_{\bar{I}^{\prime \prime}, \overline{I^{\prime \prime}}} \leq \Delta_{J^{\prime \prime}, J^{\prime \prime}} \Delta_{\overline{J^{\prime \prime}}, \overline{J^{\prime \prime}}}
$$

in products of principal $n \times n$ minors of $2 n \times 2 n$ matrices.

\section{Generalization to noncomplementary index sets}

A more general inequality than (3.8) has the form

$$
\Delta_{I, I^{\prime}} \Delta_{K, K^{\prime}} \leq \Delta_{J, J^{\prime}} \Delta_{L, L^{\prime}}
$$


That is, the pairs $(I, K)$, etc. need not be complements. However, any such inequality which holds for all totally nonnegative matrices can be deduced from Theorem 3.2 or equivalently from Corollary 3.4. We will give a combinatorial proof of this fact using the families of graphs introduced in Section 2.

Let $p$ and $p^{\prime}$ be the cardinalities of $I \cup K$ and $I^{\prime} \cup K^{\prime}$, and let $q$ and $q^{\prime}$ be the cardinalities of $I \cap K$ and $I^{\prime} \cap K^{\prime}$. Necessarily, $p+q=p^{\prime}+q^{\prime}=|I|+|K|$. Applying Observation 1.3, we may interpret the product of minors $\Delta_{I, I^{\prime}} \Delta_{K, K^{\prime}}$ of any totally nonnegative matrix to be the weighted sum of path families $\pi=\left(\pi_{1}, \ldots, \pi_{p+q}\right)$ in a planar network which connect sources indexed by $I(K)$ to sinks indexed by $I^{\prime}\left(K^{\prime}\right)$ and in which all $S_{I}$ to $T_{I^{\prime}}$ paths $\left(S_{K}\right.$ to $T_{K^{\prime}}$ paths) are vertex-disjoint. We will say that such a path family obeys the $\left(I, I^{\prime}, K, K^{\prime}\right)$ crossing rule. The following neccessary condition for eight sets to satisfy (4.1) for all totally nonnegative matrices was first stated in [3, Prop. 2.2].

Observation 4.1 Let $I, I^{\prime}, J, J^{\prime}, K, K^{\prime}, L, L^{\prime}$ be subsets of $[m]$. Unless $I \cup K$ and $J \cup L$ are equal as multisets, and $I^{\prime} \cup K^{\prime}$ and $J^{\prime} \cup L^{\prime}$ are equal as multisets, the products $\Delta_{I, I^{\prime}} \Delta_{K, K^{\prime}}$ and $\Delta_{J, J^{\prime}} \Delta_{L, L^{\prime}}$ are incomparable as functions on totally nonnegative matrices.

Proof: Suppose $i$ is an index which appears with greater multiplicity in $I \cup K$ than in $J \cup L$, and let $G$ be any planar network of order $m$ in which the unique edge leaving source $s_{i}$ has weight $c$. If $c$ is large enough, then the weighted path matrix of $G$ satisfies

$$
\Delta_{I, I^{\prime}} \Delta_{K, K^{\prime}}>\Delta_{J, J^{\prime}} \Delta_{L, L^{\prime}}
$$

On the other hand, if $c$ is close enough to zero, we have the opposite strict inequality. Similarly, if $I^{\prime} \cup K^{\prime}$ and $J^{\prime} \cup L^{\prime}$ are not equal as multisets, then the products are again incomparable.

Without loss of generality, we shall assume that the sets $I \cup K$ and $I^{\prime} \cup K^{\prime}$ are equal to $[p]$ and $\left[p^{\prime}\right]$, respectively. Otherwise we can delete appropriate matrix rows and columns to make this true. Necessary and sufficient conditions for eight sets to satisfy (4.1) are analogous to the inequalities (3.3) and (3.7). The appropriate choices of $I^{\prime \prime}, J^{\prime \prime}$, and $n$ are as follows. Let $n$ be the number $\frac{1}{2}\left(p-q+p^{\prime}-q^{\prime}\right)$, let $\eta$ be the unique order preserving map

$$
\eta:(I \backslash K) \cup(K \backslash I) \rightarrow[p-q]
$$

and let $\eta^{\prime}$ be the unique order reversing map

$$
\eta^{\prime}:\left(I^{\prime} \backslash K^{\prime}\right) \cup\left(K^{\prime} \backslash I^{\prime}\right) \rightarrow[p-q+1,2 n] .
$$

Define the subsets $I^{\prime \prime}$ and $J^{\prime \prime}$ of $[2 n]$ by

$$
\begin{aligned}
& I^{\prime \prime}=\eta(I \backslash K) \cup \eta^{\prime}\left(K^{\prime} \backslash I^{\prime}\right), \\
& J^{\prime \prime}=\eta(J \backslash L) \cup \eta^{\prime}\left(L^{\prime} \backslash J^{\prime}\right) .
\end{aligned}
$$


Theorem 4.2 Let $I, I^{\prime}, J, J^{\prime}, K, K^{\prime}, L, L^{\prime}$ be subsets of $[m]$, and define $p, p^{\prime}, q, q^{\prime}, n, \eta$, $\eta^{\prime}, I^{\prime \prime}$, and $J^{\prime \prime}$, as above. Then the following statements are equivalent.

1. In each totally nonnegative matrix of size at least $m \times m$, the minors $\Delta_{I, I^{\prime}}, \Delta_{J, J^{\prime}}, \Delta_{K, K^{\prime}}$, $\Delta_{L, L^{\prime}}$ satisfy

$$
\Delta_{I, I^{\prime}} \Delta_{K, K^{\prime}} \leq \Delta_{J, J^{\prime}} \Delta_{L, L^{\prime}}
$$

2. The multisets $I \cup K$ and $J \cup L$ are equal, the multisets $I^{\prime} \cup K^{\prime}$ and $J^{\prime} \cup L^{\prime}$ are equal, and the sets $I^{\prime \prime}, J^{\prime \prime}$ satisfy

$$
\max \left\{\left|B \cap I^{\prime \prime}\right|,\left|B \backslash I^{\prime \prime}\right|\right\} \geq \max \left\{\left|B \cap J^{\prime \prime}\right|,\left|B \backslash J^{\prime \prime}\right|\right\}
$$

for each even subinterval $B$ of $[2 n]$.

Proof: $\quad(2 \Rightarrow 1)$ Suppose (1) is false. Then there exists a planar network in which more path families obey the $\left(I, I^{\prime} K, K^{\prime}\right)$ crossing rule than obey the $\left(J, J^{\prime}, L, L^{\prime}\right)$ crossing rule. This network contains a subnetwork $G$ which is a union of $(p+q)$ paths from $p$ sources to $p^{\prime}$ sinks with the property that more path families $\pi=\left(\pi_{1}, \ldots, \pi_{p+q}\right)$ which cover $G$ obey the $\left(I, I^{\prime}, K, K^{\prime}\right)$ crossing rule than obey the $\left(J, J^{\prime}, L, L^{\prime}\right)$ crossing rule.

Applying the procedure defining $\phi$ to $G$, we obtain a graph in which exactly $n$ connected components are paths whose endpoints belong to the $2 n$-element set

$$
S_{I \backslash K} \cup S_{K \backslash I} \cup T_{I^{\prime} \backslash K^{\prime}} \cup T_{K^{\prime} \backslash I^{\prime}}
$$

By the discussion following Corollary 2.2, these $n$ paths define a perfect matching of $S_{(I \backslash K)} \cup T_{\left(K^{\prime} \backslash I^{\prime}\right)}$ with $S_{(K \backslash I)} \cup T_{\left(I^{\prime} \backslash K^{\prime}\right)}$, which is not a perfect matching of $S_{(J \backslash L)} \cup T_{\left(L^{\prime} \backslash J^{\prime}\right)}$ with $S_{(L \backslash J)} \cup T_{\left(J^{\prime} \backslash L^{\prime}\right)}$. Let $H$ be the graph in $\mathcal{G}_{3}(2 n)$ realizing this matching, in which vertex $i(1 \leq i \leq p-q)$ corresponds to the source in $S_{I \backslash K} \cup S_{K \backslash I}$ with the $i$ th smallest index and vertex $j\left(p-q<j \leq p-q+p^{\prime}-q^{\prime}\right)$ corresponds to the sink in $T_{I^{\prime} \backslash K^{\prime}} \cup$ $T_{K^{\prime} \backslash I^{\prime}}$ with the $j$ th greatest index. Let $B=\left[b_{1}, b_{2}\right]$ be a minimal interval of [2n] such that $\left(b_{1}, b_{2}\right)$ is an edge of $H$ and $b_{1}, b_{2}$ both belong to $J^{\prime \prime}$ or both belong to $\bar{J}^{\prime \prime}$. Then we have

$$
\max \left\{\left|B \cap I^{\prime \prime}\right|,\left|B \backslash I^{\prime \prime}\right|\right\}<\max \left\{\left|B \cap J^{\prime \prime}\right|,\left|B \backslash J^{\prime \prime}\right|\right\} .
$$

( $1 \Rightarrow 2$ ) Let $B=\left[b_{1}, b_{2}\right]$ be a minimal subinterval of [2n] which satisfies (4.2), and let $j_{1}$ and $j_{2}$ be the preimages of these numbers with respect to the maps $\eta$ and/or $\eta^{\prime}$. Create a graph $H=(V, E)$ as follows.

1. Place $2 p+2 q$ vertices on a horizontal line.

2. Define six sets of symbols

$$
\begin{aligned}
S & =\left\{s_{i} \mid i \in(I \backslash K) \cup(K \backslash I)\right\}, \\
U & =\left\{u_{i} \mid i \in(I \cap K)\right\},
\end{aligned}
$$




$$
\begin{aligned}
U^{\prime} & =\left\{u_{i}^{\prime} \mid i \in(I \cap K)\right\}, \\
T & =\left\{t_{i} \mid i \in\left(I^{\prime} \backslash K^{\prime}\right) \cup\left(K^{\prime} \backslash I^{\prime}\right)\right\}, \\
V & =\left\{v_{i} \mid i \in\left(I^{\prime} \cap K^{\prime}\right)\right\}, \\
V^{\prime} & =\left\{v_{i}^{\prime} \mid i \in\left(I^{\prime} \cap K^{\prime}\right)\right\} .
\end{aligned}
$$

3. Label the leftmost $p+q$ vertices by the $p+q$ symbols $S \cup U \cup U^{\prime}$, in order of nondecreasing indices. Label the rightmost $p+q$ vertices by the $p+q$ symbols $T \cup V \cup V^{\prime}$, in order of nonincreasing indices.

4. Connect each of the $q+q^{\prime}$ pairs of the form $\left(u_{i}, u_{i}^{\prime}\right)$ or $\left(v_{i}, v_{i}^{\prime}\right)$ by $q+q^{\prime}$ noncrossing arcs.

5. Connect the $b_{1}$ st (from the left) singleton to the $b_{2}$ nd (from the left) singleton by an arc.

6. Draw $p+q-1$ more arcs above the vertices to complete a noncrossing perfect matching of $S_{I \backslash K} \cup T_{K^{\prime} \backslash I^{\prime}}$ with $S_{K \backslash I} \cup T_{I^{\prime} \backslash K^{\prime}}$.

The arc drawn in step (5) prevents $H$ from inducing a perfect matching of $S_{J \backslash L} \cup T_{L^{\prime} \backslash J^{\prime}}$ with $S_{L \backslash J} \cup T_{J^{\prime} \backslash L^{\prime}}$. Now consider the planar network $G$ which is obtained from $H$ by creating $\rho(H)$ and then identifying all pairs of vertices of the form $\left(u_{i}, u_{i}^{\prime}\right)$ or $\left(v_{i}, v_{i}^{\prime}\right)$. There are no path families in $G$ which obey the $\left(J, J^{\prime}, L, L^{\prime}\right)$ crossing rule, but there are some which obey the $\left(I, I^{\prime}, K, K^{\prime}\right)$ crossing rule. This contradicts (1).

Example 4.1 Consider the two products of minors

$$
\Delta_{\{1,2,3,6\},\{1,2,4,5\}} \Delta_{\{3,4\},\{2,5\}}, \quad \Delta_{\{1,3,6\},\{1,2,5\}} \Delta_{\{2,3,4\},\{2,4,5\}} .
$$

We claim that these products are incomparable. To see this, let the map $\eta:\{1,2,4,6\} \rightarrow$ $\{1,2,3,4\}$ preserve order, and let the map $\eta^{\prime}:\{1,4\} \rightarrow\{5,6\}$ reverse order. Then define

$$
\begin{aligned}
I^{\prime \prime} & =\{\eta(1), \eta(2), \eta(6)\} \cup \emptyset \\
& =\{1,2,4\}, \\
J^{\prime \prime} & =\{\eta(1), \eta(6)\} \cup\left\{\eta^{\prime}(4)\right\} \\
& =\{1,4,5\} .
\end{aligned}
$$

Since the sets $I^{\prime \prime}, J^{\prime \prime}$ satisfy

$$
\begin{aligned}
& \max \left\{\left|[4,5] \cup I^{\prime \prime}\right|,\left|[4,5] \backslash I^{\prime \prime}\right|\right\}<\max \left\{\left|[4,5] \cup J^{\prime \prime}\right|,\left|[4,5] \backslash J^{\prime \prime}\right|\right\}, \\
& \max \left\{\left|[5,6] \cup I^{\prime \prime}\right|,\left|[5,6] \backslash I^{\prime \prime}\right|\right\}>\max \left\{\left|[5,6] \cup J^{\prime \prime}\right|,\left|[5,6] \backslash J^{\prime \prime}\right|\right\},
\end{aligned}
$$

the products of minors are incomparable.

\section{Open problems}

Note that the results of this paper reduce the problem of comparing minors in totally nonnegative matrices to the problem of counting unweighted path families in planar networks. 
This is curious, since Theorem 1.2 does not guarantee that for every totally nonnegative integer matrix $A$, there exists a planar network $G$ such that $A$ counts unweighted paths in $G$. This suggests the following open problem.

Problem 5.1 Characterize the totally nonnegative integer matrices $A=\left[a_{i j}\right]$ for which there exists a planar network $G$ such that each entry $a_{i j}$ counts unweighted paths in $G$ from source $s_{i}$ to $\operatorname{sink} t_{j}$.

Other possibilities for extending the present work are the following.

Problem 5.2 Let $P(n)$ be the poset whose elements are set partitions of $[2 n]$ into two blocks of size $n$, ordered by $I-\bar{I} \leq_{P(n)} J-\bar{J}$ whenever the inequality

$$
\Delta_{I, I} \Delta_{\bar{I}, \bar{I}} \leq \Delta_{J, J} \Delta_{\bar{J}, \bar{J}}
$$

holds for all totally nonnegative matrices. Find a simple description for $P(n)$.

Problem 5.3 Characterize the inequalities in products of $k$ minors which are satisfied by all totally nonnegative matrices, for $k>2$.

\section{Acknowledgments}

I would like to thank Sergey Fomin, John Stembridge, Eric Babson, Misha Gekhtman, Francesco Brenti, Alex Postnikov, and Harm Derksen for many helpful discussions.

\section{References}

1. F. Brenti, "Combinatorics and total positivity," J. Combin. Theory Ser. A 71 (1995), 175-218.

2. C.W. Cryer, "Some properties of totally positive matrices," Lin. Alg. Appl. 15 (1976), 1-25.

3. S.M. Fallat, M.I. Gekhtman, and C.R. Johnson, "Multiplicative principal-minor inequalities for totally nonnegative matrices," Adv. Appl. Math. (2002).

4. S. Fomin and A. Zelevinsky, "Total positivity: Tests and parametrizations," Math. Intelligencer (2001), $23-33$.

5. I. Gessel and G. Viennot, "Binomial determinants, paths, and hook length formulae," Advances in Mathematics 58 (1985), 300-321.

6. I. Gessel and G. Viennot, "Determinants and plane partitions," 1989. Preprint.

7. S. Karlin and G. McGregor, "Coincidence probabilities," Pacific J. Math. 9 (1959), 1141-1164.

8. B. Lindström, "On the vector representations of induced matroids," Bull. London Math. Soc. 5 (1973), 85-90.

9. C. Loewner, "On totally positive matrices," Math. Z. 63 (1955), 338-340.

10. G. Lusztig, "Total positivity in reductive groups," in Lie Theory and Geometry: In Honor of Bertram Kostant, vol. 123 of Progress in Mathematics. Birkhäuser, Boston, 1994, pp. 531-568.

11. R. Stanley, Enumerative Combinatorics, vol. 2. Cambridge University Press, Cambridge, 1999.

12. A. Whitney, "A reduction theorem for totally positive matrices," J. d'Analyse Math. 2 (1952), 88-92. 